\title{
Conventional Coronary Artery Bypass Graft Surgery
}

\author{
Dominic Emerson ${ }^{1,2}$, Gregory Trachiotis ${ }^{2,3}$ \\ ${ }^{1}$ Resident in Surgery, Georgetown University, Washington DC, USA \\ ${ }^{2}$ Division of Cardiothoracic Surgery and Heart Center, Veterans Affairs Medical Center, Washington DC, USA \\ ${ }^{3}$ The George Washington University Medical Center, Washington DC, USA \\ Email: gregory.trachiotis@va.gov
}

Received 3 February 2016; accepted 24 April 2016; published 27 April 2016

Copyright (C) 2016 by authors and Scientific Research Publishing Inc.

This work is licensed under the Creative Commons Attribution International License (CC BY).

http://creativecommons.org/licenses/by/4.0/

(c) (1) Open Access

\begin{abstract}
Coronary artery bypass grafting (CABG) remains one of the most common procedures worldwide, and remains the gold standard therapy for symptomatic coronary artery disease (CAD) that involves the left main coronary artery, triple vessel CAD with or without left ventricular dysfunction, and other complex coronary CAD not amenable to percutaneous intervention, especially in diabetic patients or those with chronic total coronary occlusion. The majority of CABG operations are performed utilizing cardiopulmonary bypass, though beating heart or off-pump strategies have gained popularity by some surgeons. This review focuses on current technical strategies, and advances in conduit harvest and utility, and fundamental management components of cardiopulmonary bypass and beating heart principles.
\end{abstract}

\section{Keywords}

\section{Cardiopulmonary Bypass, Beating Heart, Mini-Circuit}

\section{Introduction}

In the 1980s and 1990s, clinical studies showed that coronary artery bypass graft (CABG) surgery is the gold standard therapy for patients with multivessel coronary artery disease [1] [2]. The procedure is safe, provides reliable relief of angina, and is associated with improved long-term survival. Indications for CABG surgery have changed with continued growth of interventional cardiology capabilities. Primary indications for CABG surgery currently include left main stenosis greater than 50\%, multivessel coronary disease (particularly in patients with left ventricular dysfunction or insulin-dependent diabetes), and percutaneous coronary intervention failures. 
In more recent years, much attention has been focused on the development of minimally invasive CABG techniques, particularly off-pump coronary artery bypass surgery. Stabilizing platforms, coronary shunts, and positioning devices have contributed to the adoption of these techniques. Nonetheless, conventional CABG surgery using cardiopulmonary bypass (CPB) under cardioplegic arrest remains the preferred approach in most centers today. The key points regarding the performance of conventional CABG surgery are emphasized here, as well as some recent advance in CPB techniques.

\section{Conduits}

Multiple conduit options exist for CABG surgery. The most commonly used conduits include the left internal mammary artery (LIMA) and greater saphenous vein [2]-[4]. Total arterial revascularization (TAR) has recently gained further attention, and may be facilitated using the right internal mammary artery (RIMA), radial artery, or right gastroepiploic artery [3] [5] [6]. TAR can typically be achieved using bilateral IMA combined with other arterial conduits, and has been shown to significantly reduce the incidence of graft atherosclerosis and late cardiac related events, such as angioplasty, reoperation, or death. It should be considered in all young patients $(<50$ years old) with critical stenosis (greater than $70 \%$ ). Nonetheless, utilizing careful harvest techniques and preparation to minimize conduit trauma, current evidence demonstrates that a LIMA to the LAD, and SVG to other territories provides excellent long-term symptom control and survival in most patients [1] [2].

\subsection{Internal Mammary Artery}

It is well established that use of the LIMA is associated with excellent long-term patency ( $95 \%$ at 15 years), improved survival, and decreased late cardiac events after CABG surgery. In traditional CABG, the LIMA is harvested through a full sternotomy after opening the left pleural space. The conduit is mobilized with a 2-cm pedicle containing the mammary veins, transversus thoracis muscle, and fascia. The endothoracic fascia is divided, and side branches are clipped or tied as they are encountered. The LIMA is harvested from its bifurcation distally to the subclavian vein proximally. We use topical and injection of papaverine to the pedicled IMA to dilate and preserve endothelial integrity of the artery. If the IMA needs further dilation or flow augmentation gentle use of a $1 \mathrm{~mm}$ intracororany probe followed by intraluminal papaverine can be used. If the LIMA is not long enough to reach the site of anastomosis, its length is increased by incising the fascia in several places, and dividing the mammary veins. Harvesting a skeletonized LIMA by leaving most of the endothoracic fascia in situ is utilized when a long LIMA graft is anticipated, or when bilateral mammary harvesting is performed, to help preserve sternal vascularization. Although the LIMA is the most common conduit choice, relative contraindications include 1) proximal left subclavian artery stenosis, 2) history of chest wall radiation or radical mastectomy, 3) iatrogenic fascial hematoma during harvesting, 4) emergency operation in the setting of hemodynamic decompensation, and 5) insufficient flow due to small size or persistent spasm.

\subsection{Right Internal Mammary Artery}

Most authors employ a similar harvesting strategy for the RIMA. We prefer a skeletonized harvest of the RIMA to help preserve sternal vascularization. There is increasing evidence that a second IMA graft leads to lower of morbidities, including death (see above). Several clinical studies have demonstrated long-term patency of the pedicled RIMA to be comparable to that of the LIMA. However, the patency rate of the free RIMA has been reported to be lower, primarily due to the difficult anastomosis between the RIMA and the ascending thoracic aorta. We find that a skeletonized RIMA can often reach distal coronary arteries, and does not need to be a free graft. Our preference is to use a RIMA that is T-grafted off the LIMA to lateral wall vessels [5].

\subsection{Radial Artery}

The use of the radial artery as a conduit for CABG has recently increased after being largely abandoned due to high failure rates. The midterm patency rates are encouraging ( $85 \%$ to $90 \%$ at 5 years), but are clearly inferior to IMA grafts, and are similar to of SVG grafts in recent studies [5]-[7].

Harvesting the radial artery begins with evaluation of the presence of an intact palmar arch (Allen's test) by placing a pulse oximeter on the thumb and compressing the ulnar and radial arteries until loss of signal. The ulnar artery is released, and one looks for the presence of the pulse oximetry waveform. If Allen's test is negative 
(i.e., there is an intact ulnar artery), the radial artery is safe to harvest. A curvilinear incision is made over the radial artery just proximal to the wrist crease extending to the proximal forearm slightly lateral to midline. After identification of the radial artery, a confirmatory intraoperative Allen's test is performed by occluding the proximal radial artery and feeling for a retrograde pulse distally. The length of the pedicle is exposed, and branches are clipped. Special care must be taken to avoid the lateral antebrachial and superficial radial nerves. During the harvest a solution consisting of verapamil, nitroglycerin, heparin, and sodium bicarbonate in lactated Ringer's solution is used topically to prevent vasospasm. After harvest, the artery is flushed and soaked with this solution. Additionally, Verapamil, $0.5 \mathrm{mg} / \mathrm{hr}$, is given to patients intravenously if they are hemodynamically able to tolerate it. Postoperatively the patient is started on amlodipine, $5 \mathrm{mg} /$ day, or Diltiazam SR 60 - $120 \mathrm{mg} /$ day after discontinuing intravenous verapamil [5] [7].

\subsection{Right Gastroepiploic Artery and Inferior Epigastric Artery}

Use of the right gastroepiploic artery and inferior epigastric artery has been described, but their application is limited. Several reports have presented a good patency rate in the early postoperative period, and a patency rate of $80 \%$ has been reported for the right gastroepiploic artery at 5 years. Utilization of these vessels is limited to situations where other conduit options simply do not exist in a given patient.

\subsection{Saphenous Vein}

The greater saphenous vein was the first conduit used for CABG surgery. Despite current trends towards TAR, 95\% of current procedures still employ the use of at least one venous conduit. Advantages of venous conduits include simplicity, flexibility for sequential anastomosis, and reliable high early flow as they are not prone to spasm. In the long-term, the well described development of atherosclerotic lesions in saphenous venous conduits adversely affects patency, which has been shown to be around $50 \%$ at 10 to 12 years, but this trend in improving with better harvest techniques (especially open versus endoscopic), handling and preservation of the vein, and better choices of distal target application (author's experience) [1] [4] [8].

Harvesting the GSV has evolved since its inception to include endoscopic harvest techniques. Using an endoscopic dissector (several on the market), the GSV is harvested through small $(2 \mathrm{~cm})$ incisions, rather than the traditional incision which can run the length of the leg. This technique has reduced significantly the morbidity of leg wounds by reducing leg wound infection rate to less than $1 \%$, and allowing earlier patient mobilization. There is some continued debate as to the long-term effects on graft patency of this harvesting technique with both in-vivo and in-vitro analysis, as well as, a its cost. In our practice, with the newer and less traumatic EVH equipment, we have incorporated EVH as the standard, yet still maintain attention to atraumatic harvest, a buffered bath containing papaverine for SVG bath, and minimal stretching during preparation. When performing an open harvest approach, we use with minimal tissue trauma or distension, papaverine and/or nitroglycerin buffered bath, and rarely do we have to harvest above the knee; the annual wound complication rate is $<1 \%$.

\section{Operative Principles}

\subsection{Management of the Ascending Aorta}

Embolization of atheroma from the ascending aorta or the proximal aortic arch during cannulation or crossclamping is the principal cause of stroke after CABG surgery and one of the major causes of morbidity [9]. Due to the rising incidence of severe atherosclerotic lesions of the ascending aorta, safe cannulation techniques have become increasingly important. In addition to preoperative screening, we routinely perform intraoperative TEE and digital palpation of the aorta. Patients who are identified to have aortic plaque then undergo intraoperative epiaortic scanning to characterize the burden of aortic disease. We currently use the VeriQ system (MediStim USA), which has an easy to use probe, the use of which adds only a minimal amount of time to the case. If aortic disease is absent or minimal, CPB with cardioplegic arrest can be performed safely. However, in the presence of significant aortic disease (multiple or circumferential calcification, significant luminal thickening, or mobile plaque), alternative cannulation sites (arch, femoral, or axillary) or procedures (see below) to conventional CABG surgery that avoid aortic cross-clamping must be considered [10]. If aortic cannulation can be performed safely, yet that are problem zones for proximal anastomosis, SVG graft can be anastomosed to the innominate artery, or use of a proximal aortic device, and some resurgence of T-graft off an IMA. 


\subsection{Cardiopulmonary Bypass}

After the selection of cannulation sites, an appropriate dose of heparin is administered (400 units $/ \mathrm{kg}$ ) with a target activated clotting time (ACT) > 400 seconds (target 480 seconds). Following anticoagulation, mammary conduits can be divided and examined for flow. Purse string sutures are placed at the cannulation sites in the right atrial appendage and aorta. A soft cannula $(7.0 \mathrm{~mm}[21 \mathrm{Fr}]$ or $8.0 \mathrm{~mm}[24 \mathrm{Fr}]$, THI Aortic Cannulae Straight, Kendall Argyle USA) is placed in the aorta, and a two/three-stage venous cannula (29/37 Fr X $37 \mathrm{~cm}$, Edwards Lifesciences LLC) is placed into the right atrium for return. Retrograde and aortic root cardioplegia and/or vent catheters can be placed as case mandates, or at surgeon's discretion. Prior to commencement of CPB, we perform retrograde autologous priming (RAP), which reduces conventional 2 - 3 liters of prime to 200 - 300 cc of Plasmalyte or albumin only. We believe that RAP can reduce the systemic inflammatory response syndrome (SIRS) of CPB (see Mini Circuit section), and maintains coagulation factors [11]. CBP is then initiated and, if adequate, ventilation is ceased. Cold (4 degree $\mathrm{C}$ ) antegradecardioplegia is administered as cross-clamping is preformed for myocardial protection. Cardioplegia is four parts blood to one part cardioplegia (Our cardioplegia is $1 \mathrm{~L}$ Plegisol, $100 \mathrm{mg}$ Lidocaine, $10 \mathrm{~g}$ mannitol, $11 \mathrm{mEq}$ sodium bicarbonate, and $40 \mathrm{mEq}$ potassium). The arresting dose includes potassium at a concentration of $30 \mathrm{mEq} / \mathrm{liter}$; and a maintenance dose is 11 $\mathrm{mEq} / \mathrm{L}$ of potassium with the other components at the same concentration. Cardioplegia is typically given in an antegrade fashion $(10 \mathrm{ml} / \mathrm{kg})$, however when retrograde doses are required, $300 \mathrm{ml}$ of cold cardioplegia is also given at induction, and the maintenance cardioplegia every 20 minutes. Maintenance of CPB is performed at flows calculated for cardiac indices between 1.8 - 2.4. Patient mean pressure are maintained at $60 \mathrm{~mm} \mathrm{Hg}$, and $>70 \mathrm{mmHg}$ for patients with renal, peripheral or cerebrovascular disease and age $>70$ years. We maintain core (bladder) temperature at $\geq 35 \mathrm{C}$ (tepid) while on pump, and rewarming can begin as soon as the last anastomosis is started. For patients with acute coronary syndrome, myocardial infarction, or significant myocardial ischemia, a warm blood dose (400 - $500 \mathrm{ml}$ at $36 \mathrm{C}$ ) with be given via the antegrade or retrograde cannulae.

\subsection{Mini Pump Circuit (Miniaturized CPB)}

While CABG with CPB remains the gold standard, CPB itself is a source of potential morbidity due to the systemic inflammatory response syndrome (SIRS) which occurs following CPB. Attempts to reduce the deleterious effects SIRS has recently led to the development of minimized circuits. The goal of these mini-cardiopulmonary bypass circuits (MCPBC) has been to minimize the contact between blood and foreign material, as this contact has been proposed as the inciting event that triggers SIRS in the patient. MCPBC are closed circuit devices that serve the same purpose as traditional CPB, with several important changes: MCPBCs have no open venous reservoir-eliminating air-blood contact, tubing length is reduced to lower required priming volume, tubing is or can be heparinized, and cardiotomy suction is eliminated. These circuits are typically also easier to use and maintain. Early experience with these systems has demonstrated reduction in SIRS-associated sequlae, and reduction in transfusion requirements [11]. Due to this, it is currently our practice to utilize MCPBCs in all CPB procedures (which includes elimination of cardiotomy suction). Once RAP is achieved, our circuit can facilitate all cardioplegia and vent lines, and can be employed for ALL types of cardiac procedures. Additional techniques use for blood conservation include use of a hemoconcentrator, cell saver, and returning all "blood pump" back to patient; with these techniques and utilizing MCPBC on all cardiac cases intraoperative blood usage is less than $16 \%$ in my practice (STS reports currently at about $50 \%$ ).

\subsection{Grafting Technique}

Distal anastomosis is undertaken first, followed by proximal anastomosis. After inspection and palpation of the desired distal target, an arteriotomy is made distal to obstructive lesion and then extended with coronary scissors. The conduit is then trimmed and beveled, ensuring an appropriate lie and an opening $25 \%$ larger than the arteriotomy. 7 - 0 or 8 - 0 prolene is used in a continuous fashion for all distal anastomosis, with small bites taken around the toe and heel, and always with the bites on the coronary artery being inside to out. For thick walled or plaques in the coronary artery, especially in the LAD, a 7'0 Everpoint needle (Prolene, Ethicon INC) facilitates easier passage of the needle for anastomosis. If an endarterectomy is needed, it is performed and the conduit is grafted either directly to the coronary artery or to a vein hood attached to the coronary artery. In general venous grafts are performed first, starting from the right system, then proceeding to the left. If lesion sets are proximal 
with $>70 \%$ obstruction, we prefer to use sequential venous anastomosis to left or right sided territories; and rarely do our sequential anastomosis cross territories (i.e.: PDA of right to OM of left). IMA to LAD is constructed last as the patient is rewarmed. Deairing of IMA grafts is performed by first allowing forward bleeding into the LAD prior to securing the suture line. Sequential grafting to more than one artery with the LIMA is occasionally required when there is a shortage of conduits, but we generally avoid this in our practice.

Before performing proximal anastomosis conduit length of the SVG is again assessed. A punch device is utilized to create a $4 \mathrm{~mm}$ hole in the aorta at the site of planned anastomosis while on CPB and a partial clamp generally used. Venous proximal conduit anastomosis are created with 5 - 0 prolene. If a free LIMA is required it is sewn to a portion of the proximal SVG graft with 7'0 prolene. Grafts are then deaired (using a 25 G needle for veins). Distal targets are inspected. Patients are then weaned from CPB and cannulae are removed.

Alternative techniques to Conventional CPB: Beating Heart On-Pump (BHOP) and Off-Pump Cardiopulmonary Bypass (OPCAB)

The advent of cardiac surgery was made possible by the creation of CPB to obtain a bloodless and stationary operating field. However, in instances where traditional CPB with cross-clamping is not a viable option, beating heart on-pump (BHOP) and off-pump CABG (OPCAB) can be utilized. In addition to limiting aortic manipulation and associated sequelae, I have found these techniques have been associated with decreased transfusion requirements and a reduced systemic inflammatory response syndrome (SIRS) response, which is responsible for much of the noncardiac morbidity after open-heart surgery using CPB.

BHOP uses the mini-pump circuit as described above, yet no cardioplegia is administered, and all revascularization takes place after usual cannulation of the ascending aorta and right atria on an empty beating heart [11]-[13]. Coronary anastomosis is facilitated utilizing the same techniques and stabilizers used in OPCAB (see below). I have found this technique extremely usual in patients with large hearts, those that have sustained a recent MI with myocardial edema, and in stunned hearts with a low ejection fraction. Avoiding cross-clamp, and reperfusion injury under these scenarios can be advantageous.

Should CABG be not indicated or allowed with conventional CPB or mini-circuit techniques, OPCAB can be an alternative technique for complete coronary revascularization. OPCAB can be technically more challenging for the surgeon and team. As such, communication with the anesthesia team and ensuring that they are comfortable with the cardiopulmonary physiology and hemodynamic swings that can occur during manipulation of the heart is essential. The initial approach for OPCAB is similar to traditional CABG, with a median sternotomy my preferred approach and heparinization to an ACT of $>300$ seconds (heparin dose 1/2 conventional CPB). After harvesting of appropriate conduit (typically LIMA, with or without RIMA or other conduit as needed), planned revascularization should occur in order of increasing cardiac manipulation (i.e., anterior wall, then inferior wall, then lateral wall). Proper positioning of the heart is facilitated by using currently available stabilizing tools (we use an Apical suction device and Octopus Evolution coronary stabilizer, Medtronic INC) are key features to OPCAB success. We also use intraoperative TEE to assess left ventricular outflow tract (LVOT) to gauge right heart physiology, and the VeriQ system (MediStim USA) to assess graft patency with real time transit time flow analysis and Doppler duplex imaging. (This device has also features to identify intramyocardial vessels for on or off pump bypass) [12] [13]. We generally limit the $\mathrm{T}$ incision in the pericardium, and do not need deep pericardial stay sutures or require to open the right pleural space for cardiac positioning and visualization for complete revascularization. To facilitate coronary visualization silastic vessel loops are placed proximally and distally but NOT circumferentially near the arteriotomy. To further optimize visualization, the field is kept free of blood with a humidified $\mathrm{CO}_{2}$ blower during the anastomosis. LIMA to LAD is uniformally constructed first to restore anterior wall blood flow. This generally facilitates improved cardiac performance and myocardial manipulation for inferior and lateral targets. If occlusion of the target causes unacceptable hemodynamic changes, intracoronary shunting may be performed using a commercially available shunt. OPCAB is used in $>80 \%$ of my revascularization practice.

\section{Conclusion}

Current mortality rates for CABG using CPB are between $1 \%$ and $3 \%$. This is despite the fact that the patient population referred for CABG surgery is increasingly older, with worse cardiac disease, more comorbidities, and potential later referral due to earlier attempts at medical and/or percutaneous management. Utilizing a minicircuit for all CPB cardiac cases has helped reduce blood transfusion rate and SIRS in our practice. Also, when 
necessary, applying alternative CPB techniques, like BHOP or OCPAB (which should be part of a cardiac surgeon and team's armamentarium) and will certainly help manage an aging and more challenging patient population, and may provide adjuvant CPB techniques to emerging technologies like Hybrid revascularization, TAVI or TEVAR or Mechanical Assist Device implant. As the surgical techniques for coronary revascularization continue to advance, and standardized adjuvant risk factor medical management becomes prevalent, patients will continue to see further benefits [13] [14]. We anticipate that CABG surgery will remain the gold standard for coronary disease for the foreseeable future.

\section{References}

[1] Jones, E.L., Craver, J.M., Guyton, R.A., Bone, D.K., Hatcher, C.R. and Riechwald, N. (1983) Importance of Complete Revasculaization in Performance of the Coronary Artery Bypass Operation. The American Journal of Caridology, 51, 7-12. http://dx.doi.org/10.1016/S0002-9149(83)80003-4

[2] Leavitt, B.J., et al. (2001) Use of the Internal Mammary Artery Graft and In-Hospital Mortality and Other Adverse Outcomes Associated with Coronary Artery Bypass Surgery. Circulation, 103, 507. http://dx.doi.org/10.1161/01.CIR.103.4.507

[3] Lytle, B.W., et al. (1999) Two Internal Thoracic Artery Grafts Are Better than One. The Journal of Thoracic and Cardiovascular Surgery, 117, 855. http://dx.doi.org/10.1016/S0022-5223(99)70365-X

[4] Tyras, D.H., Barner, H.B., Kaiser, G.C., Codd, J.E., Penninton, D.G. and Williams, V.L. (1980) Bypass Grafts to the Left Anterior Descending Coronary Artery: Saphenous Vein Versus Internal Mammary Artery. The Journal of Thoracic and Cardiovascular Surgery, 3, 327-333.

[5] Barner, H.B., Bailey, M., Gutherie, T.J., Pasque, M.K., Moon, M.R., Damiano, R.J., et al. (2012) Radial Artery Free and T Graft Patency as Coronary Artery Bypass Conduit over a 15-Year Period. Circulation, 126, S140-S144. http://dx.doi.org/10.1161/CIRCULATIONAHA.111.081497

[6] Tatoulis, J., et al. (1999) Total Arterial Coronary Revascularization: Techniques and Results in 3220 Patients. The Annals of Thoracic Surgery, 68, 2093-2099. http://dx.doi.org/10.1016/S0003-4975(99)01150-9

[7] Patel, A., Asopa, S. and Dunning, J. (2006) Should Patients Receiving a Radial Artery Conduit Have Post-Operative Calcium Channel Blockers? Interactive Cardiovascular and Thoracic Surgery, 5, 251-257. http://dx.doi.org/10.1510/icvts.2006.129262

[8] Motwani, J.G. and Topol, E.J. (1998) Aortocornary Saphenous Vein Graft Disease: Pathogenesis, Predisposition, Prevention. Circulation, 97, 916-931. http://dx.doi.org/10.1161/01.CIR.97.9.916

[9] Wareing, T.H., et al. (1992) Management of the Severely Atherosclerotic Ascending Aorta during Cardiac Operations. The Journal of Thoracic and Cardiovascular Surgery, 103, 453.

[10] Aranki, S.F., et al. (1994) Single-Clamp Technique: An Important Adjunct to Myocardial and Cerebral Protection in Coronary Operations. The Annals of Thoracic Surgery, 58, 296-303. http://dx.doi.org/10.1016/0003-4975(94)92196-2

[11] Trachiotis, G.D., Baldwin, D., Zena, E. and Greenberg, M. (2008) Early Experience with Miniaturized Cardiopulmonary Bypass (Mini-Pump) in Cardiac Surgery. Interactive Cardiovascular and Thoracic Surgery, 7, 103.

[12] Trachiotis, G.D., Zena, E. and Greenberg, M. (2008) Transesophageal Echocardiograpghy for Monitoring Left Ventricular Outflow Tract during Beating Heart Surgery: Hemodynamic Implications. Interactive Cardiovascular and Thoracic Surgery, 7, S98.

[13] Huddleston, T. (2012) Operative Strategies in Acute Coronary Syndrome- Techniques and Secondary Prevention. In Tech Publications, Acute Coronary Syndromes, Chapter 12.

[14] Trachiotis, G.D. (2010) Dual Antiplatelet Therapy after CABG: A Calculated Benefit (Review). Innovations, 5, 317-325. 